\title{
LA INTERVENCIÓN DE LA FUNDACIÓN ROCKEFELLER EN LA CREACIÓN DE LA SANIDAD CONTEMPORÁNEA EN ESPAÑA (*)
}

\author{
Esteban Rodríguez Ocaña \\ (*) Trabajo realizado dentro del Proyecto DGICYT PB94-0813. Agradezco las facilidades dadas por el Dr. Darwin \\ Stupleton, director, y el personal del Rockefeller Archive Center (North Tarrytown. N.Y., EE.UU.). Igualmente, las noti- \\ cias suministradas por Josep Lluis Barona sobre Charles Bailey y Gustavo Pittaluga, obtenidas del mismo Archivo.
}

En anteriores ocasiones ante este mismo foro ${ }^{1,2}$, he tenido ocasión de señalar que la formación de la Salud Pública española, pese a contar con una legislación similar a las de las naciones europeas vecinas, registró la particularidad de sufrir graves deficiencias en dotación y en personal capacitado. Señalaba entonces que las acciones modernizadoras aparentemente más eficaces tuvieron un motor exterior, en forma de compromiso o intervención internacional: es el caso de la adopción de la clasificación internacional de causas de muerte, en 1900, la ordenación de la estadística militar, en particular después de 1903, las medidas de sanidad portuaria entre 1909 y 1912, o el inicio del subsidio de maternidad como primer paso hacia el establecimicnto dcl seguro social (conclusiones de la Conferencia de Washington de 1919, refrendadas por España en 1922). Como concluía en mi intervención en el II Encuentro Marcelino Pascua ${ }^{2}$ y confirman estudios recientes, ${ }^{3}$ la Salud Pública se desarrolló en Europa dentro de contextos ideológicamente conciliadores, tales como la unificación nacional (Alemania, Italia), la doctrina del solidarismo (Francia) o la campaña por la eficiencia nacional (Gran Bretaña, Suecia). Mas como desde 1917 el escenario político interno discurrió por la

Correspondencia:

Departamento de Historia de la Ciencia.

Facultad de Medicina. Universidad de Granada.

Avda. de Madrid, 11.

18012 Granada.

Correo electrónico: erodrig@ugr.es. senda de la confrontación, no hubo ocasión para que la modernización sanitaria se alzara, en España, con un puesto preeminente en el horizonte de las preocupaciones sociales.

El estudio de las relaciones entre la Fundación Rockefeller y España subraya ambos factores - las deficiencias internas y la importancia de la acción internacional- de manera relevante, además de aportarnos un punto de vista nuevo al proceso de constitución de la Sanidad pública española, como es la existencia de una auditoría permanente a lo largo de la etapa primorriverista. La organización sanitaria hispana a mediados de los años 20 era un espantajo de papel y latón y los esfuerzos para darle contenido se sustentaron sobre una alianza entre determinados círculos elitistas dentro de España y una potencia internacional, la Junta (o División) Sanitaria Internacional, International Health Board (Division) (en adelante, I.H.B.), órgano de la mencionada Fundación, que tuvo la dirección del proceso. Mostrarlo es el objeto del presente trabajo.

I. La intervención norteamericana en la Gran Guerra se acompañó del interés de dicha Fundación por Europa ${ }^{4-7}$. Pero mientras que podemos interpretar su relación con Francia o Gran Bretaña por ser países aliados durante la guerra, y con los estados centroeuropeos como forma de creación de un «cordón sanitario» para preservar Occidente de los diversos «contagios» ruso-soviéticos, la situación periférica 
de nuestro país en ambos contextos no nos permite adelantar ninguna hipótesis concreta al respecto. Sólo la inmanente tentación imperial de la I.H.B., tal vez subrayada por el antiguo papel hispano de metrópoli respecto de las Américas, explicaría el último porqué de los acuerdos entre la Fundación Rockefeller y España.

El motivo inmediato residió en las gestiones del Secretario Permanente de la Junta para la Ampliación de Estudios e Investigaciones Científicas (J.A.E.), José Castillejo (18771946), entre 1919 y $1922^{8}$. La relevancia cobrada por Estados Unidos como país receptor de pensionados de la J.A.E. por mor de la contienda, reconocido en las Memorias de la J.A.E. correspondientes a los años 1916 y 1917 (pp. XI-XII) y 1920 y 1921 (p. XIII) y cuantificado por Baratas y Fernández ${ }^{9}$, se sumó a las noticias sobre la proyección internacional de la Fundación, que pasó a ser vista como posible fuente de apoyo para sus proyectos de renovación. Existe mención a los efectos positivos de la participación sanitaria norteamericana en Centroamérica y Francia en la carta que Santiago Ramón y Cajal, como presidente de la J.A.E. dirigió al general Primo de Rivera, el 11 de diciembre de 1923 (reproducida por Sánchez Ron ${ }^{10}$ ), para solicitar una definición favorable del Directorio a la puesta en marcha de los acuerdos con las agencias Rockefeller. El sabio hispano aprovechaba la ocasión no sólo para resaltar el buen hacer de la Fundación norteamericana, sino para reafirmar la necesidad de la continuidad de la J.A.E., pues «[1a Fundación] daría a España su auxilio y guía con tal de hallar aquí una coordinación que, por su seriedad, permanencia y eficacia, garantizara el fruto de aquella generosidad».

El momento decisivo para la consecución de tales acuerdos tuvo lugar en febrero de 1922, cuando una delegación de la I.II.B., encabezada por su director, Wickliffe Rose, visitó España ${ }^{8}$. El viaje sirvió para anudar una red de contactos con círculos cualificados, designados como intelligent people en los informes internos de la Fundación, entre los que destacaron Castillejo y Gustavo Pittaluga Fattorini (1876-1955), así como para estable- cer las bases estratégicas de la intervención norteamericana ${ }^{11}$.

La documentación conservada en los Archivos Rockefeller muestra la fácil sintonía que alcanzaron Castillejo y Rose, tanto en lo tocante a fines (garantizar una dirección profesional al proceso de modernización necesario para España) como a los medios (cl programa trazado nació de las coincidencias entre lo que Castillejo solicitó y lo que la I.H.B. hacía por sistema). Recordemos que el objetivo de la Junta para la Ampliación de Estudios e Investigaciones Científicas era la renovación del mundo científico español principalmente a través de la capacitación de personas, que debían ser las portadoras de los nuevos métodos y técnicas capaces de dar sentido a las necesarias modificaciones institucionales $y$ legislativas. La Fundación, por su parte, buscaba implantar el estilo científico y la dinámica de gestión norteamericanos a través, igualmente, de la formación postgraduada de quienes, en sus países de origen, debían hacerse cargo de la dirección de la administración y la docencia. En uno como en otro caso se confiaba en el valor de irradiación o de fermento de progreso de esos profesionales nuevos, capaces de mostrar a la opinión pública y a las minorías dirigentes las ventajas de la ciencia a través de la trasformación de su entorno laboral inmediato. Así, Castillejo solicitó a la I.H.B. que efectuara una intervención local ejemplar a partir de la cual, escribió textualmente, the nation will see ${ }^{12}$, en coincidencia perfecta con la línea marcada por Wickliffe Rose al frente de la I.H.B. que proponía como método de trabajo la realización de demonstrations localizadas con el mismo objetivo ${ }^{13}$.

El contacto Pittaluga, por su parte, fue no menos significativo, en particular para los diferentes enviados temporales de la Fundación en España. Su formación y ejercicio profesional como «médico de laboratorio», su renombre internacional y su empuje y estilo personal de trabajo, en particular al frente de la Comisión central antipalúdica (desde 1920), le granjearon las mayores simpatías por parte de los sanitarios norteamericanos. Constan en los archivos de la Fundación Rockefeller las opiniones favorables de Brumpt, el catedrático de 
Parasitología de París, y del bioquímico de la Escuela de Salud Pública de la Johns Hopkins, Elmer V. MacCollum ${ }^{14}$. De hecho, fue considerado como «el hombre mejor preparado de la Sanidad española», y «el más cercano» a los puntos de vista de los enviados a España por la Fundación ${ }^{15}$. El delegado permanente llegó a decir de Pittaluga que sería el mejor Director general de Sanidad, y que «[...] seguramente lo será cuando cambie el gobierno» ${ }^{16}$, ya que tenía el inconveniente de no ser adicto al régimen primorriverista ${ }^{17}$. De la lucha antipalúdica se advirtió, en seguida, que era lo único que funcionaba de la sanidad española ${ }^{14,18}$. Su nombramiento como Director de la Escuela Nacional de Sanidad en 1930 fue considerado un triunfo.

A las buenas relaciones de Pittaluga con la División sanitaria internacional de la Fundación Rockefeller contribuyó también su participación en algunos de los proyectos internacionales indirectamente auspiciados por aquella. Por ejemplo, formó parte del Comité Permanente de la Oficina de Higiene de la Sociedad de Naciones, que recibía importante provisión de fondos de la Fundación Rockefeller, y de sus Comisiones de Paludismo y Enseñanza de la Higiene; también presidió la Conferencia Internacional de Sanidad Rural de junio de $1931^{19,20}$. Pero, no se piense que le sirvieran para conseguir un cheque en blanco; así, en 1925 se le rechazó una solicitud de apoyo financiero para un estudio sobre etiología del Kalaazar infantil en España ${ }^{21}$, o en 1926 se le denegaron ayudas para erigir una «Escuela de Enfermedades Tropicales» y para estudiar los mosquitos en el delta del Ebro ${ }^{22}$. Incluso, una vez exiliado como consecuencia de la guerra civil, no consiguió visado para entrar en Estados Unidos, bien que se lo habían ofrecido reiteradamente unos años antes ${ }^{23}$.

El acuerdo surgido del viaje de Rose a España se aprobó por la Comisión ejecutiva de la I.H.B. el 23 de mayo de 1922, oficialmente a solicitud del Gobierno español. ${ }^{24}$ Se contemplaban dos tipos de actuaciones distintas: la Junta Sanitaria enviaría algún personal técnico a España, a la vez que facilitaba becas para formación de posgrado en Salud Pública a titulados españoles.
II. Los expertos norteamericanos que llegaron a residir en España a consecuencia de este acuerdo fueron Charles A. Bailey, fogueado en la lucha contra la anquilostomiasis, y el malariólogo Rolla B. Hill, que vino durante la República. Nos referiremos con detalle sólo a la actuación del primero.

Charles Bailey perteneció al Cuerpo de Sanidad de Estados Unidos, antes de pasar al servicio de la Fundación Rockefeller entre 1916 y 1941. Sirvió inicialmente en El Salvador, y antes de venir a España realizó el curso completo de capacitación en Salud Pública de la Escuela de la Universidad Johns Hopkins. A nuestro país llegó con la misión inmediata de realizar un estudio epidemiológico de la anquilostomiasis. A la vez, como encargo confidencial, Bailey debía estudiar la situación general de la sanidad española para evaluar el alcance que deberían tener futuras posibles intervenciones ${ }^{25}$. Residió en España entre diciembre de 1924 y junio de 1926, y se siguió ocupando de los asuntos españoles, con frecuentes viajes, desde París, al menos hasta 1929. Su gestión resultó muy cficiente, se ganó el respeto y la confianza de las autoridades y de los círculos cercanos a Castillejo y a Pittaluga, a quienes, a su vez, respetó y admiró sin ambages. La Memoria de la J.A.E. para los cursos 1924-25 y 1925-26, publicada en Madrid en 1927, transmitía el siguiente juicio: «El tacto y la pericia del doctor Bailey han conseguido allanar todo género de obstáculos, y su conocimiento del país lo coloca en condiciones de prestarnos inapreciables servicios» (p. 177). No es de extrañar que, de manera consecutiva, sus jefes norteamericanos tuvieran en alto aprecio su trabajo ${ }^{26}$.

La opinión de Bailey sobre los dirigentes y empleados de la Sanidad española, sin embargo, fue negativa, comenzando por la persona de Francisco Murillo Palacios (1865-1944), el Director general de Sanidad entre 1923 y 1928, a quien caracterizó reiteradamente como un auténtico obstáculo ${ }^{27-30}$. Y su diagnóstico de situación sobre el estado de la sanidad española no fue menos lapidario: despilfarro, ineficencia, desastre organizativo, despreocupación respecto a los problemas de salud de la población, tales eran las notas que la caracteri- 
zaban, en un contexto laboral donde la escasa dedicación se daba la mano con los bajos niveles salariales. Sintéticamente lo expresó así en marzo de 1926: "The whole public health service is fundamentally wrong, literally one might say almost non-functioning ${ }^{15}$.

Además de llevar a cabo su tarea inicial sobre la anquilostomiasis, Bailey redactó un informe sobre la sanidad española, en abril de 1926, hasta la fecha inédito ${ }^{31}$, del cual se derivaron otras acciones inmediatas, según la Memoria de la J.A.E. correspondicnte a los cursos 1924-25 y 1925-26 (Madrid, 1927: 176), a saber: un refuerzo del programa de becas para personal de la Dirección General de Sanidad (D.G.S.) y la cooperación con la campaña antipalúdica. Otros efectos a más largo plazo de la actividad de Bailey tuvieron que ver con la reforma de los servicios centrales sanitarios así como la intervención en la provincia de Cáceres, en forma de programa integral de asistencia preventiva, que se llevó a cabo a partir de 1929 . Sus proyectos de intervención municipal, sin embargo, fueron rotundos fracasos, tanto en Madrid ${ }^{32}$, como en Má$\operatorname{laga}^{33,34,28}$.

Como plasmación inmediata del acuerdo, antes incluso de la llegada de Bailey, se produjo la dotación de tres becas, otorgadas a Joaquín de Prada, Donato Fuejo García y Antonio Ortiz de Landázuri para estudios en Estados Unidos durante el curso 1923-24. Los dos primeros se especializaron en laboratorio sanitario, mientras que el tercero lo hizo en salud pública general y administración sanitaria. La adjudicación de becas sufrió una parálisis de un año, similar a la consecutiva al golpe militar en la vida de la J.A.E., pero, entre 1925 y 1930, amparó a otras 21 personas, 19 médicos, de los cuales se especializaron 14 en salud pública general, cuatro en laboratorio y uno en estadística, además de un ingeniero sanitario y una enfermera. Entre 1931 y 1936 se dotaron otras 20 becas, 15 para mujeres, una matrona y las restantes para enfermeras de salud pública, y 5 en médicos varones, tres para administración sanitaria y dos para laboratorio ${ }^{35}$. Esta distinta composición del personal obedece a las prioridades marcadas por la Rockefeller: durante el primer quinquenio, refuerzo del centro sanitario y de la lucha antipalúdica; durante el segundo, la creación de la enfermería de salud pública ${ }^{36}$.

Existieron varios modelos de beca, en función de su duración, entre 4 y 24 meses. Los agentes de la Rockefeller preferían las estancias más largas, advertidos de la dificultad con el inglés que presentaban la mayoría de los candidatos. La estancia típica se dividía en tres partes consecutivas: estudio del idioma, curso en la Escuela de Salud Pública de Johns Hopkins en Baltimore y viajes por distintos centros sanitarios del Este y Sur de los Estados Unidos, así como europeos, en su caso, cuando se trataba de becas de dos años. Las becas de cuatro y seis meses se concedían, casi siempre, para estancias en centros europeos, y la mayoría lo fueron en relación con la lucha antipalúdica. La duración de la beca estaba prefigurada, en muchos casos, según el puesto que ocupaba el becario en la sanidad española, de forma que cuanto más cercano al Director general, menos tiempo se les concedía. A Ortiz de Landázuri, a Sadí de Buen (ambos, de la D.G.S.) o a José Alberto Palanca (inspector provincial de Sanidad en Madrid) no se les permitió estar más de seis meses alejados de sus responsabilidades, bien que los dos primeros consiguieron sendas prórrogas que les permitieron completar sus progamas de trabajo. En cambio, a Francisco Ruiz Morote, inspector provincial en Cáceres, Marcelino Pascua o Antonio del Campo Cardona, estos dos sin vinculación con la administración sanitaria, aunque aspiraban a tenerla a su regreso, se les permitió sin problemas cumplir dos años de formación entre Estados Unidos y Europa.

En la selección de becarios, Bailey registró diversos momentos tensos frente a Murillo, el máximo responsable sanitario del momento, uno de cuyos rasgos más nefastos, en opinión del enviado norteamericano, consistía en su aversión casi patológica a permitir que sus subordinados lo superasen en cualquier sentido ${ }^{29}$. Muy pronto, convencido de la inconsistencia de las oficinas centrales de la sanidad española, Bailey se decantó por encontrar un estadístico, un ingeniero sanitario y un epidemiólogo de cara a la posible reorganización de la D.G.S. ${ }^{32}$. A mediados de 1925, contaba con cinco candidatos, Sadí 
de Buen, Marcelino Pascua, Antonio Ortiz de Landázuri (que quería repetir) Emilio Luengo y Ramón Montalbán García-Noblejas, un ingeniero de caminos que, luego de conseguir los parabienes de ambas partes, renunció por haber obtenido plaza de ingeniero provincial en Teruel ${ }^{37}$. La candidatura de De Buen tuvo que superar la oposición de Murillo, con quien se había enfrentado en unas oposiciones para cubrir el puesto de Director del Hospital de Infecciosos, aunque a costa de ver reducido su permiso a sólo seis meses ${ }^{38}$. Lo mismo ocurrió con Ortiz, del que Bailey conocía de antemano la oposición de Murillo a permitir que volviera a marcharse de España, y que tampoco agradaba al norteamericano, que planteó su caso sólo como elemento de negociación para conseguir el permiso para De Buen. La trayectoria de Ortiz, «el único sanitario español con una formación práctica» resultado de su beca Rockefeller, producía en Bailey una gran perplejidad, como se puso de manifiesto en el intento de actuación higiénica en Málaga. La D.G.S. accedió, inicialmente, a conceder a Ortiz una comisión de servicio, con sueldo completo, durante dos años, para que dirigiera la nueva dependencia malagueña ${ }^{28}$. Cuando el plan fracasó, al retirar Murillo el apoyo gubernativo, Bailey hasta se alegró, dudoso de que hubiera salido bien de todas formas por tener que contar obligadamente con aquel ${ }^{15}$.

Una condición fundamental para la concesión de la ayuda era la garantía por parte gubernamental de que, al regreso, el becario o becaria trabajaría en un puesto en relación con la formación adquirida. De esta forma, la Fundación se preservaba del uso meramente curricular y turístico de las becas e intentaba conseguir una infiltración eficaz de sus protegidos en los organigramas locales. Bailey, escarmentado por el bajo rendimiento de la inversión en los tres primeros becarios (de los que escribió que, un año después de su regreso, «no habían hecho nada por mejorar sus respectivos servicios» ${ }^{32}$ ) y cuya elección había correspondido a las autoridades españolas, se esforzó por encontrar personas mejor dispuestas, que debían firmar un compromiso de retorno a España una vez terminada su beca, a la vez que buscó las mayores garantías por parte de las autoridades para asegurar el mejor puesto a los becarios a su regreso. Si bien pretendió conseguir el apoyo expreso del Ministro, incluso del mismo Rey, en la práctica se tuvo que conformar con la palabra del Director general de Sanidad, en forma de escrito (statement) que acompañaba a las solicitudes de beca.

Pese a la garantías arrancadas por escrito, el problema del empleo eficiente de lo becarios se convirtió en otro frente de batalla entre Bailey y la D.G.S. Recordemos que, en este punto, se producía una total sintonía entre los deseos de los patrocinadores norteamericanos y los de la Junta para Ampliación de Estudios, la garante de los acuerdos sanitarios: para que cualquier cambio positivo tuviera lugar, era preciso contar de antemano con las personas preparadas. Así lo manifestaba, en un contexto nacional, Ramón y Cajal en 1918: «[...] no iremos más deprisa de lo que permita el personal de cuya eficiencia podamos responder, factor esencial imposible de improvisar ${ }^{39}$, y 10 corroboraba el presidente de la I.H.B. ante su enviado en España en 1925, a quien estimulaba a seleccionar sólo aquellos becarios sobre los que hubiera garantías de que podrían desarrollar a su vuelta un trabajo eficaz, como se hacía con éxito en otros países ${ }^{40}$. Desde esta perspectiva, la Fundación norteamericana se trazó una línea de actuación sumamente pragmática: sólo se subvencionaría la especialización y las personas que contaran con el visto bueno de los responsables políticos españoles, aunque eso supusiera dejar fuera a «jóvenes prometedores» que no fueran del agrado del Director general de Sanidad. A su vez, Bailey planteó que sólo al regreso de los becarios sería posible organizar verdaderas actividades sanitarias en España, como se demostró con los programas provinciales de Cáceres, desde 1929, y Albacete, desde 1932.

Los becarios seleccionados con vistas a una posible reorganización de los organismos centrales sanitarios obtuvieron, con mayor o menor dificultad, los puestos previstos, mientras que otros se reintegraron a su puesto de origen con la obligación de mejorar sus comctidos (caso de los inspectores provinciales de Sanidad o los médicos de la lucha antipalúdica). Así, Ortiz de Landázuri fue nombrado jefe de la Brigada Sanitaria central al volver de Estados Unidos en 1925 , bien que su desempeño no introdujo cambio alguno en el devenir de esta, mientras que 
tanto Emilio Luengo como Marcelino Pascua tuvieron que esperar casi dos años desde su regreso para que se hiceran realidad sus respectivas asignaciones previstas (y prometidas por escrito por Murillo ante la I.H.B.), aunque Luengo tampoco cumplió con las expectativas confiadas y se dedicó a un trabajo privado más lucrativo. Bailey se quejó de que Murillo, el Director general de Sanidad, jamás llegó a reunirse con ninguno de los 14 becarios regresados antes de 1927 para interesarse por su formación y por sus proyectos ${ }^{41}$.

III. El apoyo técnico y financiero de la I.H.B. se sustentaba sobre un explícito pragmatismo apolítico. Por ese motivo, y disintiendo de lo que había sido tradición hispana - por ejemplo, según Martín Salazar, la reforma de la Sanidad debía venir «de lo alto, por iniciativa del poder público y de los legisladores ${ }^{42}$-, enfocaban la reforma de la sanidad desde actuaciones periféricas ( «era preferible llegar a las autoridades nacionales desde las provincias» antes que al contrario), lo que se podía llevar a cabo sin modificaciones legislativas ${ }^{43}$. Bien es verdad que los intentos de actuar sobre la propia D.G.S. se abandonaron muy pronto, por la falta de sintonía con Murillo, tan chirriante que el propio Bailey dejó escapar, en los momentos finales de su estancia, que dudaba que fuera posible cambio alguno positivo bajo el régimen político existente ${ }^{15}$.

El convulso proceso de gestación del Servicio de Epidemiología de la D.G.S. muestra las limitaciones del momento con nitidez y los límites del pragmatismo norteamericano. Bailey había convencido a Murillo de su necesidad a mediados de 1926. Le explicó, por ejemplo, que era necesario detectar el origen de las decenas de casos de fiebre tifoidea que, con carácter mortal, se producían cada mes en Madrid, a fin de conseguir su disminución y eventual desaparición, actuando sobre los focos y las cadenas infecciosas. Al regreso de Estados Unidos de Emilio Luengo, ayudante de Parasitología en el Instituto Nacional de Higiene y en el laboratorio de la Facultad de Medicina de Madrid, le propuso convertirlo en jefe del nuevo servicio, que se dotaría con dos de los tres inactivos componentes de la Brigada Sanitaria Central. La I.H.B. contribuiría durante cinco años a completar la dotación económica de dicha sección, suplementando el sueldo del jefe de la misma para que pudiera ejercer con dedicación completa ${ }^{44}$. Unos meses después, Murillo contraofertó con la reconversión de la antedicha Brigada, postura que no encontró la simpatía de Bailey ${ }^{29}$, pero salió adelante por la voluntad de aquel, quien proponía nombrar a Luengo jefe a tiempo parcial de la misma (porque compatibilizaba su trabajo con el empleo en una compañía suministradora de aguas), transformarle el nombre, trasladarla a nuevos locales en el edificio del Alfonso XIII e instalar un nuevo laboratorio bacteriológico ${ }^{45}$. Hasta noviembre de 1927, sin embargo, no se decretaron estos cambios, que supusieron muy pocas mejoras reales. Así, en febrero de 1928 todavía Luengo no había recibido ni nombramiento ni material de trabajo alguno ${ }^{46}$, mientras que en agosto, la opinión de Bailey era que Luengo estaba demasiado ocupado con su trabajo privado para atender al oficial, de manera que desaconsejó la cooperación. Finalmente, en 1929 se puso en marcha el servicio, sin jefe, confiando los enviados norteamericanos en que Pascua se haría cargo del mismo, conjuntamente con su trabajo en Estadística ${ }^{47}$.

Otro elemento de radical novedad que aportó la intervención Rockefeller fue la exigencia de la dedicación completa al servicio público, naturalmente mejor remunerado de lo que estaba hasta la fecha (por cuyo motivo, una parte sustancial de los recursos financieros aportados se invirtieron en incrementar los sueldos, de manera que Sadí de Buen, Ruiz Morote y Marcelino Pascua se convirtieron en los primeros sanitarios a tiempo completo en la nómina gubernamental, gracias al apoyo de la Fundación). Resulta significativo que la noticia de que se había aprobado en 1926 un presupuesto extraordinario de 12 millones, a emplear en seis años, para edificaciones del ramo de Sanidad, llevara al norteamericano a recordar al ufano Murillo que ese dinero tendría mucho mejor uso si se empleara en el pago de sueldos dignos a personal responsable, que trabajara a tiempo completo para echar a andar las unidades básicas sanitarias ${ }^{48}$. El trabajo a tiempo parcial producía un estilo «terrible», que sumado a los horarios fragmentados y a la falta de preparación daba como resultado el parasitismo de lo 
público por los intereses privados. De hecho, Bailey se escandalizó ante la inexistente frontera entre la responsabilidad pública y el provecho privado que se advertía en que, por ejemplo, varios de los altos cargos del Instituto Nacional de Higiene mantuvieran un laboratorio particular de fabricación de sueros, que hacía la competencia al propio Instituto, o que Murillo, sin renunciar a la Dirección general, asumiera el cargo de jefe de la oficina de verificación de productos bioterapéuticos, siendo dueño de una empresa que los fabricaba.

La intervención norteamericana, pues, resultó decisiva para configurar los aspectos más destacados del modelo sanitario que intentó implantar la República y que, en buena medida, se mantuvo tras la guerra civil, aunque somctido a extinción por la falta de interés del régimen de Franco. La exigencia de una formación especializada, para lo que se creó la Escuela Nacional de Sanidad, el establecimiento del servicio de Estadística como columna vertebral de la D.G.S., la organización sanitaria interior según el modelo sectorizado de centros de Higiene rural ensayado en Cáccres y sancionado por la Conferencia internacional de $1931^{49}$ así como la inspiración de un régimen de incompatibilidades en el ejercicio de cargos públicos fueron consecuencias directas de la presencia de la Fundación Rockefeller. De este modo se establecieron unos horizontes estratégicos de calidad y de servicio que volvieron a conectar con los propósitos reformistas de los años setenta, tras el largo sueño a que los sometió el franquismo.

\section{BIBLIOGRAFÍA}

1. Rodríguez Ocaña, E. La estadística en la administración sanitaria española del siglo veinte. En: Las estadísticas demográfico-sanitarias. Primer Encuentro Marcelino Pascua. Madrid: Instituto de Salud Carlos III; 1992: 35-60.

2. Rodríguez Ocaña, E. La Salud Pública en España en el contexto europeo, 1890-1925. Rev San Hig Publica 1994; 68 (monográfico): 11-28.

3. Porter, D. editor. The History of Public Health and the Modern State, Amsterdam: Rodopi [Clio Medica, 26], 1994.
4. Weindling P. The Rockefeller Foundation and German Biomedical Sciences, 1920-1940: From Educational Philanthropy to International Science Policy. En: N. Rupkee editor. Science, Politics, and the Public Good. Essays in Honor of Margaret Gowing. New York: Macmillan Press; 1988: 119-40.

5. Weindling P. Public Health and Political Stabilisation: the Rockefeller Foundation in Cen tral and Eastern Europe between the Two World Wars. Minerva 1993; 31: 253-67.

6. Murard L, Zylberman, P. Les Rockefeller Medicine Men en France (1917-1923). En: Cohen JL, Damisch $\mathrm{H}$, editores. Américanjsme et modernité. L'idéal américain dans l'architecture. Paris: EHESS Flammarion; 1994: 267-82.

7. Balinska MA. The National Institute of Hygiene and Public Health in Poland, 1918-1939. Social History of Medicine 1996; 9: 427-45.

8. Rodríguez Ocaña E, Bernabeu Mestre J, Barona JL. La Fundación Rockefeller y España, 19141939. Un acuerdo para la modernización científica y sanitaria. En: García JL, Moreno JM, Ruiz G (coords.) Estudios de historia de las técnicas, la arqueología industrial y las ciencias. VI Congreso de la Sociedad Española de Historia de las Ciencias y de las Técnicas, Segovia-La Granja, 1996. Salamanca, C. de Cultura de la Junta de Castilla y León, 1998, vol. 2, pp. 531-39.

9. Baratas Díaz LA, Fernández J. Becas de ampliación de estudios en Biología y ciencias básicas de la Medicina en la España del primer tercio del siglo XX. Dynamis 1993; 13: 247-64.

10. Sánchez Ron JM. La Junta para Ampliación de Estudios e Investigaciones Científicas 80 años después. En Sánchez Ron, J. M. 1907-1987. La Junta para Ampliación de Estudios e Investigacioncs Científicas 80 años después, Madrid: CSIC; 1988. vol. 1.p. 15-6.

11. Notes on Public Health and Medical Education in Spain, Rockefeller Archive Center (en adelante, RAC), Rockefeller Foundation Archives (en adelante, RF), (Record Group) 5, (Series) 2, (Box) 60, (Folder) 392.

12. Castillejo a Rose, 6-02-1922, RAC, RF, 5, 1.2, 148, 1959.

13. Fosdick RB. The Story of the Rockefeller Foundation. London: Odham Press; 1952: 46-57.

14. Gunn a Russell, 25-11-1925, RAC, RF, 5, 1.2, 238,3059 . 
15. Bailey a Gunn, 18-03-1926, RAC, RF, 5, 1.2, $273,3464$.

16. Bailey a Russell, 2-06-1926, RAC, RF, 5, 1.2, 273,3461 .

17. Gunn a Russell, 17-11-1925, RAC, RF, 5, 1.2, $238,3059$.

18. Bailey a Everett, 12-02-1926, RAC, RF, 5, 1.2, 273,3462 .

19. Dubin MD. The League of Nations Health Organisation. En: Weindling P, editor. International health, organizations and movements, 1918-1939. Cambridge: CUP; 1995: 56-80.

20. Bernabeu Mestre J. El papel de la Escuela Nacional de Sanidad en el desarrollo de la salud pública en España, 1924-1934. Rev San Hig Publica 1994; 68 (monográfico): 65-90.

21. Bailey a Gunn, 22-06-1925, transmitido a Russell, 25-06-1925, y Russell a Gunn, 13-071925; RAC, RF, 5, 1.2, 238, 3061.

22. Informe trimestral (tercer trimestre) de Bailey, 12-11-1926 [remitido a Russell, 27-11-1926] RAC, RF, 5, 1.2, 273, 3464.

23. [Peticiones de ayuda de Pittaluga durante sus meses de exilio en Francia] RAC, RF, 1.1, 795 , 1, 8/9 (1939-1941).

24. Rose a Martín Salazar, RAC, RF, 5, 1.2, 148, 1959.

25. Request from the Government of Spain. RAC., RF, 5, 1.2, 148, 1959 (p. 4).

26. Gunn a Vincent (Presidente de la Fundación), 5 de febrero de 1929. RAC, RF, 1.1, 795, 2, 13.

27. Bailey a Russell, 10-04-1925, RAC, RF, 5, 1.2, 238,3060 .

28. Bailey a Gunn, 22-01-1926; Gunn a Bailey, 26-01-1926 [remitidas ambas a Russell, 26-011926], RAC, RF, 5, 1.2, 273, 3464;

29. Bailey a Russell, 12-12-1926, RAC, RF, 5, 1.2, $273,3463$.

30. Bailey a Russell, 17-11-1927, RAC, RF, 5, 1.2, 313,3980 .

31. Bailey, Report on Public Health in Spain, abril 1926, RAC, RFA, 1.1, Projects, 2, 1, 795 Spain.

32. Bailey a Russell, 9-03-1925, RAC, RF, 5, 1.2, 238,3060 .

33. Bailey a Russell, 17-08-1925, RAC, RF, 5, 1.2, 238,3061
34. Bailcy a Gunn, 4-01-1926; Gunn a Russell, 801-1926 y 28-06-26; Gunn a Bailey, 26-011926, RAC, RF, 5, 1.2, 273, 3464

35. The Rockefeller Foundation. Directory of Fellowship Awards for the years 1917-1950. Nueva York: The Rockefeller Foundation, 1950

36. Bernabeu Mestre J, Gascón Pérez E. El papel de la enfermería en el desarrollo de la salud pública española (1923-1935). La visitadora sanitaria. Dynamis 1995; 15: 151-76.

37. Gunn a Russell, 8-12-1925, RAC, RF, 5, 1.2, 238,3059 .

38. Bailey a Russell, 25-07-1925, RAC, RF, 5, 1.2, 238,3061 .

39. Ramón y Cajal al Vicepresidente del Segundo Congreso Universitario catalán, 12 de abril de 1918. En: Sánchez Ron JM. La Junta para Ampliación de Estudios e Investigaciones Científicas 80 años después, en Sánchez Ron coordinador (1988): 28-30.

40. Russell a Bailey, 7-08-1925, RAC, RF, 5, 1.2, 238,3061 .

41. Gunn a Russell, 3-09-1926, RAC, RF, 5, 1.2, 273,3464 .

42. Martín Salazar M. La Sanidad en España. En: Discursos leidos ante la Real Academia de Medicina en la recepción pública del $\mathrm{llmo}$. Sr. Doctor ... cl día 8 de junio de 1913. Madrid: Colegio de Sordomudos y Ciegos; 1913: 22.

43. Gunn a Russell, 26-08-1925, RAC, RF, 5, 1.2, 238,3959 .

44. Bailey a Gunn, 18-09-1926, RAC, RF, 5, 1.2, 273, 3464

45. Bailey a Gunn, 23-12-1926 [remitida a Russell, misma fecha], RAC, RF, 5, 1.2, 273, 3461.

46. Bailey's Diaries, Visitas a España, febrero 1928, RAC, RF, 1.1, 795, 1, 1.

47. Strode a Russell, 6-06-1930, RAC, RF, 1.1, 795K, Epidemiological Bureau 1927-1930, 3, 23.

48. Bailey a Gunn, 22-07-1926 [remitida a Russell, 30-08-1926], RAC, RF, 5, 1.2, 273, 3464.

49. Rodríguez Ocaña E. De la Junta de Sanidad al Instituto de Higiene. en: Historia y medicina en España. Homenaje al Profesor Luis S. Granjel. Valladolid: Consejería de Cultura y Turismo de la Junta de Castilla y León; 1994: 237-49. 